\title{
Publisher Correction: Multiplexed enrichment of rare DNA variants via sequence- selective and temperature-robust amplification
}

Lucia R. Wu, Sherry X. Chen, Yalei Wu, Abhijit A. Patel and David Yu Zhang

Correction to: Nature Biomedical Engineering https://doi.org/10.1038/s41551-017-0126-5; published online 4 September 2017.

In the version of this Article originally published, owing to a technical error, the Life Sciences Reporting Summary was not included; this summary is now available.

Published online: 22 November 2017

https://doi.org/10.1038/s41551-017-0156-Z 